\title{
Iron Stores in Health and Disease
}

\section{Bone-marrow Studies in 1134 Children in Punjab, India}

\author{
S. S. MANCHANDA, HARBANS LAL, and SUBHASH KHANNA \\ From the Department of Paediatrics, Medical College, Amritsar, India
}

In iron deficiency, depletion of iron stores precedes alterations in serum iron concentration, iron-binding capacity, and morphological abnormalities of red cells (Simon and Finch, 1964; Beutler, Robson, and Buttenwieser, 1958). The use of a simple and practical clinical procedure, introduced by Rath and Finch in 1948 for the semiquantitative assessment of stainable marrow iron, has enabled the clinician to diagnose this 'pre-clinical' stage of iron deficiency.

The definitive diagnosis of iron deficiency in the developing countries is particularly important because iron deficiency occurs far more frequently than hitherto suspected, and because this deficiency is often not isolated but occurs in association with other facets of malnutrition, and with infections.

The present study of 1134 subjects was undertaken to find the incidence of iron depletion in health, and to assess the prevalence of iron deficiency in various disease entities, as a basis for exploring the factors leading to the development of hyposiderosis in different clinical conditions. In addition, the comparative value of the various diagnostic parameters commonly used for diagnosing iron deficiency in clinical practice has been critically assessed.

\section{Material and Methods}

The study included 1134 children from 10 days to 14 years of age; 954 were suffering from various clinical disorders, and 180 were apparently healthy normal subjects. These 'normal' children were derived from the paediatric Follow-up-Clinic, Child Guidance Clinic, and from those convalescing from minor medical or surgical illnesses; none had suffered from any serious illness during the preceding 3 months of this study, nor had received iron medication orally or parenterally during the past 6 months. All the 1134 children under study were admitted and comprised the following five groups.

Group I-Normal (180 cases). These 180 children had $\mathrm{Hb}$ levels defined as follows (Wolman, 1957):

Received March 24, 1969. (a) $9 \mathrm{~g} . / 100 \mathrm{ml}$. in the age-group $1-9$ months; (b) $>$ $10 \mathrm{~g} . / 100 \mathrm{ml}$. in age-group 9 months to 6 years; and (c) $>11 \mathrm{~g} . / 100 \mathrm{ml}$. in age-group 6-14 years.

Group II-Malnutrition (521 cases). (a) Iron deficiency, 160 cases; (b) marasmus, 130 cases ; (c) marasmic kwashiorkor, 90 cases; (d) scurvy, 20 cases; and (e) megaloblastic anaemia, 121 cases.

Group III-Chronic systemic infections (210 cases).

Group IV-Chronic non-infective systemic illnesses (183 cases). (a) Rheumatic heart disease, 40 cases; (b) cyanotic congenital heart disease, 28 cases; (c) portal cirrhosis, 40 cases; (d) nephrotic syndrome, 62 cases; and (e) cretinism, 13 cases.

Group V-Other blood disorders (40 cases). (a) Thalassaemia, 22 cases; (b) malignancies (leukaemias), 18 cases.

After recording the history and the physical findings in each case, the routine laboratory tests included tuberculin testing, urine and stool examination, the latter particularly for helminthic/protozoal infestation, and occult blood. The selective radiological and biochemical investigations were undertaken as and when indicated for establishing the aetiological diagnosis. The haematological studies comprised $\mathrm{Hb}$ estimation (Sahli's method, the accuracy of the apparatus was checked repeatedly by the cyanmethaemoglobin method, using the photo-electric colorimeter); red cell count; haematocrit determination (Wintrobe's method, 1967); detailed survey of the Leishman's stained peripheral blood smear; calculation of red cell indices; serum iron (SI); iron-binding capacity (IBC) (Ramsay's method, 1954, 1958); unsaturated iron-binding capacity (UBIC); and percentage saturation iron-binding capacity (\% sat. IBC).

Samples of marrow ${ }^{\star}$ in children under 2 years were

* Following an inquiry by the Editors on the ethical aspects of their investigations, the authors write: 'Parental permission for undertaking the various investigations, including the "healthy normal" group, was obtained in each case. The presence of other children who had already been investigated served to allay any parental fears. No complication was met with after marrow puncture in any of the 1134 children, thus indicating the safety of this procedure.' Editor. 
aspirated from the tibia; in older children from the ilium, $2 \mathrm{~cm}$. posterior and inferior to the anterior superior iliac spine. The patients were sedated with chlorpromazine (1.5 mg./kg.), and local anaesthesia only was used.

The bone-marrow morphology was studied for qualitative abnormalities and rough quantitative assessment of cellularity, and the relative proportion of the various cellular elements. The stainable iron in the bone-marrow smears as marrow haemosiderin $(\mathrm{MH})$ was graded 0 to $3+$ according to Beutler's (Beutler et al., 1958) criteria, and the staining technique used was that of Dacie (Dacie and Lewis, 1963). The interpretation of $\mathrm{MH}$ was, however, modified so that iron deficiency was considered to be present if the $\mathrm{MH}$ content was $1+$ or less.

\section{Observations}

The final diagnosis of iron deficiency in each case was based on the absence or inadequacy of stainable iron in the marrow (i.e. graded $1+$ or less). Thus, $76 \%$ of the total 1134 children $56 \%$ of the 180 healthy ones and $81 \%$ of the 954 ill children) were iron deficient by this criterion.

The incidence of iron deficiency by the other parameters in the various clinical groups of 1134 children as compared with the inadequate $\mathrm{MH}$ content is shown in Table I. From this it is seen that there was a higher incidence of iron deficiency in the ill subjects compared to the healthy ones, the difference being reflected by all the parameters.

In Group I-amongst the 180 healthy children, $27 \%$ were aged less than 9 months, $36 \%$ were between 9 months and 6 years, and $36 \%$ were over 6 years. Though the $\mathrm{Hb}$ levels of all these cases lay within the normal ranges for their respective age-groups, $23 \%$ were judged on film appearances to be microcytic, and $12 \%$ showed hypochromia. The incidence of iron deficiency derived from other parameters (see Table IV) was $56 \%$ with inadequate $\mathrm{MH}, 22 \%$ with low SI, $29 \%$ with low percentage sat. IBC, and $36 \%$ with low MCHC.
Table II gives the incidence of stainable iron, graded quantitatively, in marrow aspirates in the different groups. Table III gives $\mathrm{Hb}$ values in 954 ill children with various clinical disorders.

Table IV shows the incidence of iron deficiency by different diagnostic parameters in 1134 children. In the 521 malnourished children of Group II, the high incidence of iron deficiency $(85 \%)$ as determined by $\mathrm{MH}$ differed little from the incidence inferred from examination of the blood film and MCHC, $77 \%$ and $73 \%$ respectively, whereas SI and percentage sat. IBC were indicative of iron deficiency in only $53 \%$ and $58 \%$ respectively.

Of the 160 children shown in Table IV, Group II (a) with nutritional iron deficiency anaemia, diagnosed on the basis of inadequate $\mathrm{MH}$ content, only $80 \%$ had hypochromic red cells, $88 \%$ low MCHC, $86 \%$ low SI, and $79 \%$ low \% sat. IBC. Thus, without marrow investigation, 12 to $21 \%$ of cases of iron deficiency were undetected by routine blood examinations, including serum iron measurements.

In the 121 cases with megaloblastic anaemia (Table IV, Group II (b)) the incidence of inadequate MH was $79 \%$, low percentage sat. IBC was $15 \%$, low SI was $23 \%$, low MCHC was $49 \%$, though $73 \%$ had hypochromic red cells. Thus the chances of missing iron deficiency in megaloblastic erythropoiesis during early infancy are minimized.

In the 210 cases with chronic infections (Table IV, Group III) $56 \%$ of which had $\mathrm{Hb}<8 \mathrm{~g} . / 100 \mathrm{ml}$., $73 \%$ showed hypochromic red cells associated with microcytosis in $43 \%$, normocytosis in $17 \%$, and macrocytosis in $12 \%$. The incidence of iron deficiency in terms of $\mathrm{MH}$ was $83 \%$.

\section{Discussion}

The high incidence of iron deficiency, $77 \%$ of the total 1134 children including $56 \%$ of the 180 healthy children, emphasizes the magnitude of iron deficiency in our child population. More than

TABLE I

Percentage of Cases Showing Iron Deficiency According to the Criteria ${ }^{\star}$ of Marrow Haemosiderin, Peripheral Blood Film Appearances, MCHC, Saturation Index, and Percentage Saturation of Iron-binding Capacity

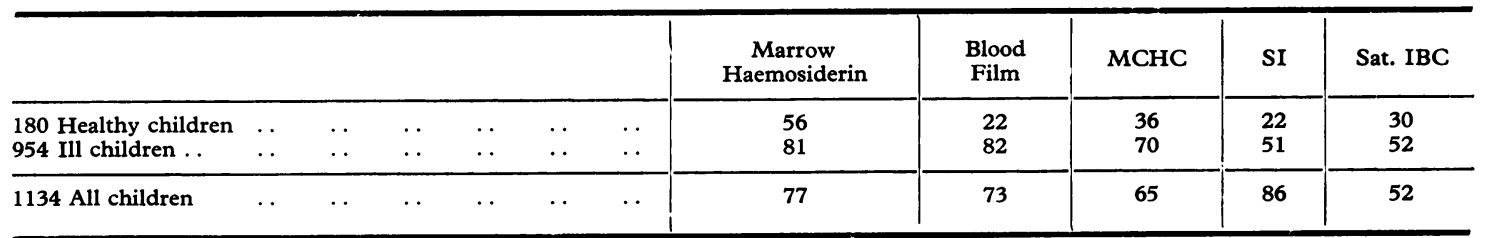

* Iron deficiency was considered to be present if marrow haemosiderin was 0 or $1+$; blood film showed hypochromic red cells; MCHC $<32 \%$ serum iron $<50 \mu \mathrm{g} . / 100 \mathrm{ml}$.; saturation of $\mathrm{IBC}<20 \%$. 
TABLE II

Grading of Marrow Haemosiderin in Different Clinical Groups

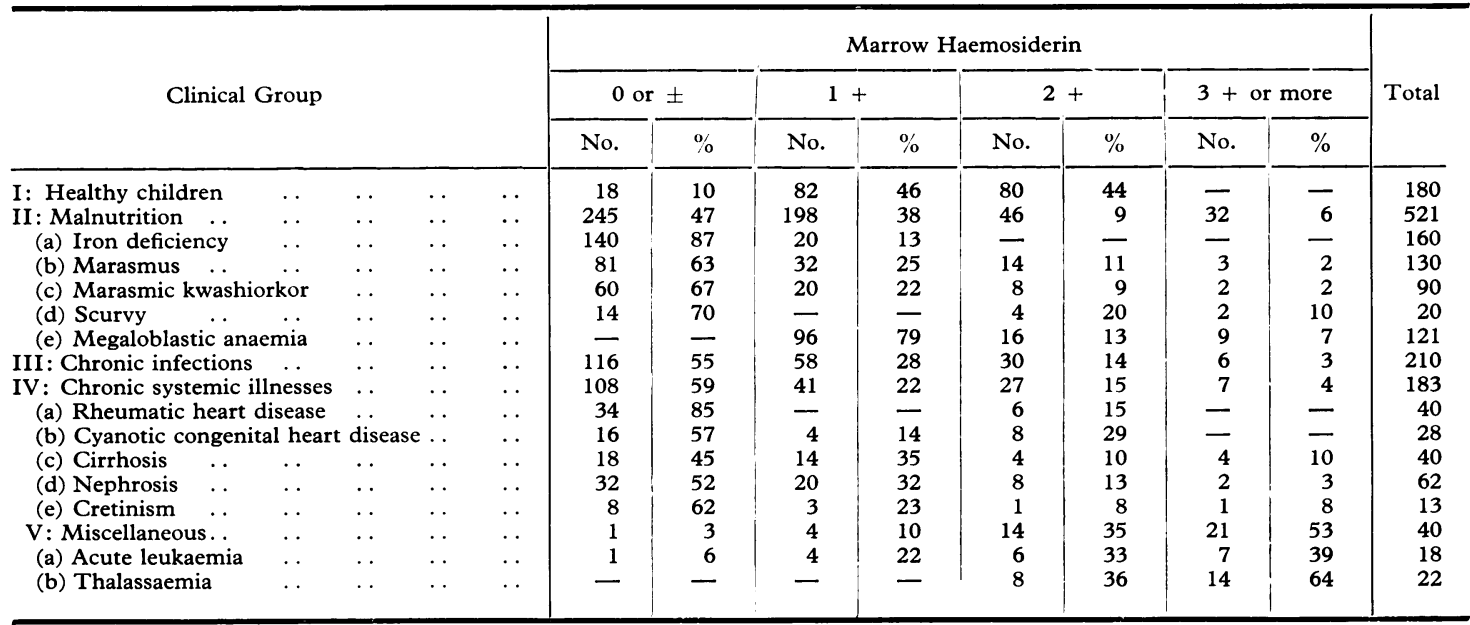

TABLE III

Haemoglobin Levels in 954 'Ill' Children

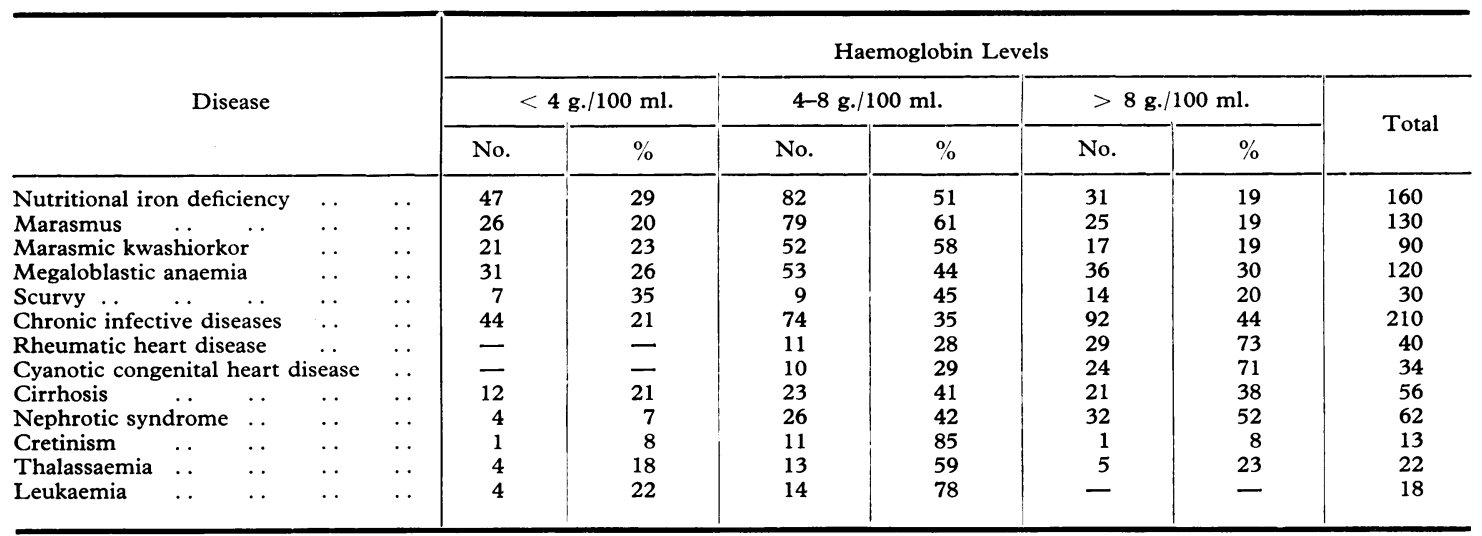

half the children in the apparently well-nourished and non-anaemic healthy group showed depleted iron stores, despite haemoglobin levels and red cell indices within the normal range, indicating a high incidence of 'pre-clinical', 'masked', or 'latent' iron deficiency. Sturgeon (1956) and Undritz (1964) made similar observations. Normal red cell indices and serum iron values fail to indicate absent or marginal iron stores. Whether amounts of storage iron, subnormal in terms of adult standards, are to be regarded as 'physiological' for the growing child, has long been debated. We consider that such marginal iron reserves are suboptimal, for owing to growth, reserves will be more quickly exhausted in the child than in the adult if there is inadequate intake, impaired absorption, or abnormal loss of iron.

If it is accepted that the most satisfactory criterion for iron deficiency is the finding of low amounts of haemosiderin in the marrow, this study has shown that the next most dependable diagnostic criterion is the presence of hypochromia of the red cells in peripheral blood films, whereas red cell indices, SI, and percentage sat. IBC are 
TABLE IV

Incidence of Iron Deficiency ${ }^{\star}$ by Different Diagnostic Parameters

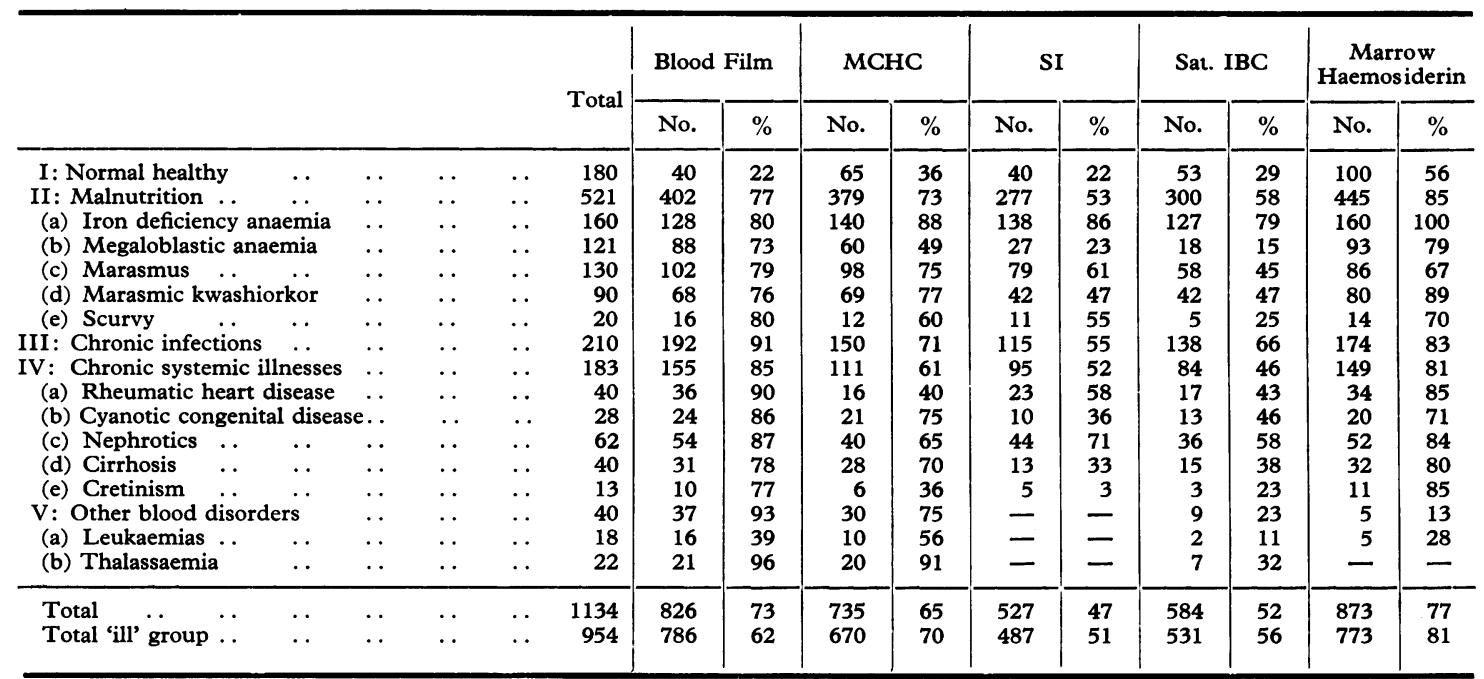

* For criteria for diagnosis of iron deficiency, see Table I.

less reliable for diagnosing iron deficiency. Cases of thalassaemia, leukaemia, and also cretinism, however, are exceptions to this generalization. We have also noted, as have others (Beutler et al., 1958), the further exception that malnutrition and infections can themselves cause hypochromic red cells, as well as alter the serum iron values, in the absence of iron deficiency as defined by marrow haemosiderin.

Protein-calorie malnutrition and anaemia have a complex interrelation which no doubt varies with the nutritional background of the different regions of the world. In the present study, iron deficiency was present in $89 \%$ cases of protein-calorie malnutrition and in $66 \%$ of those with severe calorie insufficiency. This high incidence of iron deficiency, particularly in protein-calorie malnutrition, fits in broadly with the studies reported from other tropical countries (Brown, 1965).

The high incidence $(83 \%)$ of iron deficiency anaemia in chronic infections needs comment. Chronic infections of a few weeks' duration tend to lead to normocytic-normochromic or normocytichypochromic anaemia, but not commonly to microcytosis. The degree of anaemia is usually not severe except in cases of certain severe infections, as for example, bacterial endocarditis, miliary tuberculosis (Corr, Kyle, and Bowie, 1964), or pulmonary suppuration (Wintrobe, 1967). The anaemia of infection is classically stated to be 'non iron- deficiency', despite hypoferraemia (Heilmeyer, 1964). With these probable multifactorial pathogenetic factors present in children presenting with both anaemia and infection, assessment of iron stores by $\mathrm{MH}$ estimation is particularly valuable. The high incidence of iron deficiency in the present series is no doubt partly due to pre-existing iron deficiency. The anaemia, in turn, may perpetuate or worsen the infections, so that in this part of India 'anaemia of infection' should be more appropriately termed 'anaemia with infections'.

In the third main group of systemic noninflammatory diseases, the diagnosis of iron deficiency complicating rheumatic or cyanotic congenital heart disease has practical significance, because of the possibility of relief of symptoms if these are due in part to iron deficiency. The presence of iron deficiency in cases of cyanotic polycythaemia of congenital heart disease is often overlooked, though it was present in the majority $(76 \%)$ of these cases in the present study.

\section{Summary}

Bone-marrow haemosiderin was assessed semiquantitatively in a series of 1134 children of all ages: 180 children were healthy, and the remaining 954 were suffering from a variety of disorders, mainly chronic. Iron deficiency was diagnosed if iron stores, as reflected by marrow haemosiderin, 
were low or absent. With this criterion, iron deficiency was present in $56 \%$ of the healthy children. Iron deficiency was present in $81 \%$ of all the ill children; in those with malnutrition, the incidence was $85 \%$, and in those with chronic infections, $83 \%$.

Other commonly used criteria of iron deficiency were evaluated at the same time, including red cell film appearances, red cell indices, serum iron level, and percentage saturation iron-binding capacity. Of these, the presence of hypochromia of red cells proved the most generally useful indicator of iron deficiency, while those based on serum iron levels often proved misleading.

\section{REFERENCES}

Beutler, E., Robson, M. J., and Buttenwieser, E. (1958). A comparison of the plasma iron, iron-binding capacity, sternal marrow iron and other methods in the clinical evaluation of iron stores. Ann. intern. Med., 48, 60.

Brown, R. E. (1965). Editorial. Childhood anaemia in the tropics. F. trop. Pediat., 10, 87.
Corr, W. P., Jr., Kyle, R. A., and Bowie, E. J. W. (1964). Hematologic changes in tuberculosis. Amer. F. med. Sci., 248, 709.

Dacie, J. V., and Lewis, S. M. (1963). Practical Haematology, 3rd ed., p. $85 . \quad$ Churchill, London.

Heilmeyer, L. (1964). Human hyposiderosis. In Iron Metabolism. A Symposium. Ed. by F. Gross. Springer, Berlin.

Ramsay, W. N. M. (1954, 1958). Cited by Varley, H. (1962). Practical Clinical Biochemistry, 3rd ed., p. 396. Heinemann, London.

Rath, C. E., and Finch, C. A. (1948). Sternal marrow hemosiderin. A method for the determination of available iron stores in man. F. Lab. clin. Med., 33, 81.

Simon, E. R., and Finch, C. A. (1964). Red Cell Manual. University of Washington, School of Medicine, Seattle.

Sturgeon, P. (1956). Iron metabolism. A review with special consideration of iron requirements during normal infancy Pediatrics, 18, 267.

Undritz, E. (1964). In Iron Metabolism. A Symposium. Ed. by F. Gross. Springer, Berlin.

Wintrobe, M. M. (1967). Clinical Hematology, 6th ed., p. 764 Lea and Febiger, Philadelphia. Kimpton, London.

Wolman, I. J. (1957). Laboratory Applications in Clinical Pediatrics. p. 35. McGraw-Hill, New York.

Correspondence to Dr. S. S. Manchanda, Department of Paediatrics, Medical College, Amritsar, India. 